Discrete Comput Geom 34:25-45 (2005)

DOI: $10.1007 / \mathrm{s} 00454-005-1158-2$

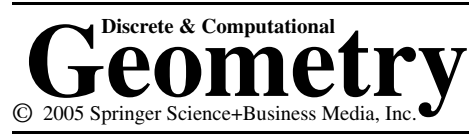

\title{
Partitions of a Polytope and Mappings of a Point Set to Facets*
}

\author{
Roman N. Karasev \\ Department of Mathematics, Moscow Institute of Physics and Technology, \\ Institutskiy per. 9, Dolgoprudny, Russia 141700
}

\begin{abstract}
Three theorems of this paper generalize previous results of the author on conjectures of A. Bezdek and V. V. Proizvolov. They show the existence of mappings from a given point set to the set of facets of a given polytope that satistfy some special conditions.

Developing the same technique, some results on convex polytope partitions are presented, two of them dealing with partitions with prescribed measures of parts. Then we prove a corollary on the existence of a possibly nonconvex polytope with a given set of vertices, containing given points in its interior.

We also consider problems of the following type: find an assignment of vectors from a given set to the parts of a given convex partition of $\mathbb{R}^{n}$ so that the shifts of the parts by their corresponding vectors either do not intersect by interior points or cover $\mathbb{R}^{n}$.
\end{abstract}

\section{Introduction}

Theorems 1-3 generalize Theorems 2-4 of [2]. They show the existence of mapping $f$ of a given finite set in $\mathbb{R}^{n}$ to the set of facets of a given polytope such that some conditions hold: for example, the convex hulls conv $F \cup f^{-1}(F)$ for different facets $F$ do not intersect pairwise by interior points.

Some of the results in [2] may be considered as a case of Theorems 1-3 when the number of points to be mapped equals the number of facets. These theorems also lead to Corollary 2 about convex partitions of $\mathbb{R}^{n}$ that generalizes the corollaries from [2]. Corollary 2 shows that for the partition of a special type, defined in this paper, one can find an assignment of vectors from a given set to the parts of this convex partition of $\mathbb{R}^{n}$ so that the shifts of the parts by their corresponding vectors either do not intersect by interior points or cover $\mathbb{R}^{n}$.

\footnotetext{
* This research was supported by the Russian Fund of Fundamental Researches Grants Nos. 03-01-00801 and 03-01-06207.
} 
Theorems 4 and 5 generalize Theorem 4 from [1] to the case of $\mathbb{R}^{n}$. They deal with the following problem: find a partition of a given polytope such that its parts have given measures and every part contains a prescribed facet of the polytope.

Now we introduce some notation. Let $V \subseteq \mathbb{R}^{n}$, then

1. conv $V$, lin $V$, and aff $V$ denote the convex, linear, and affine hull of $V$;

2. int $V$, rint $V, \operatorname{cl} V$, bd $V$, and $|V|$ denote the interior, relative interior (if $V$ is convex), closure, boundary, and number of elements of $V$;

3. $\operatorname{dim}_{l} V, \operatorname{dim}_{a} V$, and codim $V$ denote the linear dimension, affine dimension, and codimension of $V$;

4. $I_{m}=\{1,2, \ldots m\}$ denotes the set of indices and $S^{m}$ denotes the group of permutations of $I_{m}$;

5. $\operatorname{dist}(x, y)$ and $\operatorname{dist}(X, Y)$ denote the distance between points $x$ and $y$ or subsets $X$ and $Y$ of some metric space;

6. for $A, B \subseteq \mathbb{R}^{n}$ denote

$$
A+B=\{a+b: a \in A, b \in B\}, \quad A \stackrel{*}{-} B=\{x: x+B \subseteq A\},
$$

the Minkowski sum and the geometric difference of $A$ and $B$.

Let $L_{1}^{n}$ be a space of affine functions (polynomials of degree 1) on $\mathbb{R}^{n}$. Suppose $f \in L_{1}^{n}$; by definition, put

1. $H(f)=\left\{x \in \mathbb{R}^{n}: f(x)=0\right\}$-the hyperplane;

2. $H^{-}(f)=\left\{x \in \mathbb{R}^{n}: f(x)<0\right\}$ - the open halfspace;

3. $H^{+}(f)=\left\{x \in \mathbb{R}^{n}: f(x) \geq 0\right\}$ - the closed halfspace.

Recall that a polyhedral set is an intersection of a family of halfspaces

$$
X=\bigcap_{i \in I_{m}} H^{+}\left(f_{i}\right) .
$$

Suppose $X$ is a polyhedral set, denote $F_{i}=H\left(f_{i}\right) \cap X$. We assume that $\operatorname{codim} F_{i}=1$ for all $i \in I_{m}$ and define the facets of $X$ to be the sets $\left\{F_{i}\right\}_{i \in I_{m}}$.

Also, put $H_{i}=H^{-}\left(f_{i}\right)$ for all $i \in I_{m}$.

If a polyhedral set is bounded, then it is called a polytope.

Theorem 1. Let $X$ be a polytope in $\mathbb{R}^{n}$, and let $\left\{F_{i}\right\}_{i \in I_{m}}$ be its facets. Let $A \subset \mathbb{R}^{n}$, $l=|A|$, and let $\left\{l_{i}\right\}_{i \in I_{m}}$ be a set of positive integers such that $\sum_{i \in I_{m}} l_{i}=l$. Then there exists a partition of $A$ into $A_{i}\left(i \in I_{m}\right)$ satisfying the following conditions:

1. $\left|A_{i}\right|=l_{i}$ for any $i \in I_{m}$;

2. let $C_{i}=\bigcap_{a \in A_{i}} \operatorname{conv}\left(a \cup F_{i}\right)$, then sets $\left\{C_{i}\right\}_{i \in I_{m}}$ cover $X$, in other words,

$$
X \subseteq \bigcup_{i \in I_{m}} C_{i} .
$$

Theorem 1 is formulated for a bounded polytope, so it is natural to ask what can be done for an unbounded polyhedral set. This theorem is true for a given set $A$ and a 
polytope. Then we can apply a projective transformation that maps one facet to a subset of the hyperplane at infinity; note that any polyhedral set that does not contain a straight line can be obtained this way.

Theorem 1 will be true for such an unbounded polyhedral set $X$ if we consider the facet at infinity $F_{m}$ along with $m-1$ normal facets, denote

$$
I=\left\{x \in \mathbb{R}^{n}: \forall \lambda>0, \lambda x \in X-X\right\},
$$

and put $C_{m}=\cap_{a \in A_{i}}(a+I)$ (and $C_{i}$ for $i=1, \ldots, m-1$ as in Theorem 1).

See Section 6 for Corollary 2, which may be considered as a generalized form of Theorems 1 and 2 applied for polyhedral sets with "all facets at infinity".

Theorem 2. Let $X$ be a polyhedral set in $\mathbb{R}^{n}$, and let $\left\{F_{i}\right\}_{i \in I_{m}}$ be its facets. Let $A \subset X$, $l=|A|$, and let $\left\{l_{i}\right\}_{i \in I_{m}}$ be a set of positive integers such that $\sum_{i \in I_{m}} l_{i}=l$. Then there exists a partition of $A$ into $A_{i}\left(i \in I_{m}\right)$ satisfying the following conditions:

1. $\left|A_{i}\right|=l_{i}$ for any $i \in I_{m}$;

2. let $C_{i}=\operatorname{conv}\left(A_{i} \cup F_{i}\right)$, then the sets $\left\{C_{i}\right\}_{i \in I_{m}}$ do not intersect pairwise by interior points, in other words, for all $i \neq j \in I_{m}$,

$$
\operatorname{int} C_{i} \cap \operatorname{int} C_{j}=\emptyset \text {. }
$$

Theorem 3. Let $X$ be a polyhedral set in $\mathbb{R}^{n}$, and let $\left\{F_{i}\right\}_{i \in I_{m}}$ be its facets. Let $A \subset$ $\mathbb{R}^{n} \backslash X, l=|A|$, and let $\left\{l_{i}\right\}_{i \in I_{m}}$ be a set of positive integers such that $\sum_{i \in I_{m}} l_{i}=l$. Suppose that $\left|A \cap \bigcup_{i \in I} H_{i}\right| \geq \sum_{i \in I} l_{i}$ holds for all $I \subseteq I_{m}$. Then there exists a partition of $A$ into $A_{i}\left(i \in I_{m}\right)$ satisfying the following conditions:

1. $\left|A_{i}\right|=l_{i}$ for any $i \in I_{m}$;

2. let $C_{i}=\operatorname{conv}\left(A_{i} \cup F_{i}\right)$, then the sets $\left\{C_{i}\right\}_{i \in I_{m}}$ do not intersect with $X$ by interior points and do not intersect by interior points pairwise. In other words, for any $i \in I_{m}$,

$$
\text { int } C_{i} \cap \text { int } X=\emptyset \text {, }
$$

and for all $i \neq j \in I_{m}$,

$$
\operatorname{int} C_{i} \cap \operatorname{int} C_{j}=\emptyset \text {. }
$$

Theorems 2-4 from [2] may be obtained from the above theorems if $|A|=m$ and $l_{i}=1$ for all $i \in I_{m}$.

The same method allows us to prove three other theorems. The following two theorems can be considered as continuous cases of Theorem 2. Here we have a measure instead of a finite point set.

Theorem 4. Let $X$ be a polytope in $\mathbb{R}^{n}$, and let $\left\{F_{i}\right\}_{i \in I_{m}}$ be its facets. Let $\mu$ be a measure on $X$, continuous with respect to the Hausdorff metric, and let $\mu(X)=1$. Then for any set of positive numbers $\left\{\mu_{i}\right\}_{i \in I_{m}}\left(\sum_{i \in I_{m}} \mu_{i}=1\right)$ there exists a partition of $X$ into convex sets $\left\{A_{i}\right\}_{i \in I_{m}}$ such that for all $i \in I_{m}$,

$$
A_{i} \cap \mathrm{bd} X=F_{i}, \quad \mu\left(A_{i}\right)=\mu_{i} .
$$


We formulate a slightly different theorem that generalizes Theorem 4 from [1] to the case of $\mathbb{R}^{n}$ :

Theorem 5. Let $X$ be a polytope in $\mathbb{R}^{n}$, and let $\left\{F_{i}\right\}_{i \in I_{m}}$ be its facets. Let $\mu$ be a measure on $X$, continuous with respect to the Hausdorff metric, and let $\mu(X)=1$. Let $\left\{\mu_{i}\right\}_{i \in I_{m}}$ be a set of real numbers such that $\sum_{i \in I_{m}} \mu_{i}=1$ and $\mu_{i} \geq 0$ for all $i \in I_{m}$. Denote $I=\left\{i \in I_{m}: \mu_{i}>0\right\}$, then there exists a partition of $X$ into convex sets $\left\{A_{i}\right\}_{i \in I}$ such that for all $i \in I$,

$$
A_{i} \supseteq F_{i}, \quad \mu\left(A_{i}\right)=\mu_{i} .
$$

Theorem 6. Let $X$ be a polytope in $\mathbb{R}^{n}$, and let $\left\{F_{i}\right\}_{i \in I_{m}}$ be its facets. Let $V \subset$ int $X$ be a finite set and let $m \geq|V|+1$. Then there is a partition of $X$ into convex sets $\left\{A_{i}\right\}_{i \in I_{m}}$ such that for all $i \in I_{m}$,

$$
A_{i} \cap \operatorname{bd} X=F_{i}, \quad V \cap \operatorname{int} A_{i}=\emptyset .
$$

Remark. In fact, the partitions in these three theorems are affine partitions (see Section 6).

Theorem 6 leads to the following corollary:

Corollary 1. Let $W$ and $V$ be disjoint finite sets in $\mathbb{R}^{n}$, and let their union be in general position. Let $V \subset$ conv $W$, let conv $W$ have $m$ facets, and let $m \geq|V|+1$. Then there exists a possibly nonconvex polytope $Y$ with vertices $W$ such that $V \subset Y$.

Remark. In this theorem a nonconvex polytope is a bounded closed set $Y \subset \mathbb{R}^{n}$ such that bd $Y$ is a polyhedral complex, homeomorphic to $(n-1)$-dimensional sphere.

\section{Auxiliary Assertions}

Here we give some definitions and lemmas. The lemmas are taken from [2], so we do not give proofs here.

Definition. We say that the set $V$ in a linear (affine) space $L(A)$ is in general position and write $V \in L G P(V \in A G P)$ if

$$
\operatorname{dim}_{l} U=\min \left\{|U|, \operatorname{dim}_{l} L\right\} \quad\left(\operatorname{dim}_{a} U=\min \left\{|U|-1, \operatorname{dim}_{a} A\right\}\right)
$$

for any finite $U \subseteq V$.

Definition. Suppose $\mathcal{F}$ is a family of maps from the same finite set $I$ to $L(A)$, then we say that the map $\lambda \in \mathcal{F}$ is in general position and write $\lambda \in L G P(\mathcal{F})(\lambda \in A G P(\mathcal{F}))$ if

$$
\operatorname{dim} \lambda(U)=\max _{\lambda^{\prime} \in \mathcal{F}} \operatorname{dim} \lambda^{\prime}(U)
$$

for all $U \subseteq I$. 
If the family $\mathcal{F}$ is an irreducible algebraic variety, considered as a subset of $L^{I}\left(A^{I}\right)$, then $L G P(\mathcal{F})(A G P(\mathcal{F}))$ is open and everywhere dense in $\mathcal{F}$.

Definition. A polyhedral set $S \subset \mathbb{R}^{n}$ is called simple if either it is a simplex or $S=\bigcap_{i \in I_{m}} H^{+}\left(f_{i}\right)$, where $\left\{f_{i}\right\}_{i \in I_{m}} \in L G P$ and $m \leq n$.

Let $a \neq b \in \mathbb{R}^{n}$, denote by $[a b],(a b),\langle a b\rangle$, and $[a b\rangle$ the segment between $a$ and $b$, its relative interior, the straight line passing through $a$ and $b$, and the ray, emanating from $a$, passing through $b$. Also put

$$
\langle a b\rangle=[a b\rangle \backslash\{a\} \backslash(a b) .
$$

This is the ray in $\langle a b\rangle$, emanating from $b$ and not containing $a$.

Definition. A set $U \supseteq V$ is called $V$-starshaped if for any $u \in U$ and $v \in V$ we have $[u v] \subseteq U$. Clearly, if

1. $V \neq \emptyset$, then a $V$-starshaped set $U$ is starshaped;

2. sets $U_{i}, i \in I$, are $V$-starshaped, then $\bigcup_{i \in I} U_{i}$ and $\bigcap_{i \in I} U_{i}$ are $V$-starshaped;

3. $U$ is $V$-starshaped and $W \subseteq V$, then $U$ is $W$-starshaped.

Lemma 1. Let $X$ be a polyhedral set in an affine space $L$, and let $\left\{F_{i}\right\}_{i \in I_{m}}$ be its facets. Then there exists an affine embedding of $L$ into an affine space $L^{\prime}$ of larger dimension and a simple polyhedral set $S$ in $L^{\prime}$ such that $X=S \cap L$, int $X=$ (int $\left.S\right) \cap L$, and for any $i \in I_{m}, F_{i}=G_{i} \cap L$, where $\left\{G_{i}\right\}_{i \in I_{m}}$ are the facets of $S$. Also, if $X$ is a polytope, then $S$ is a simplex.

Lemma 2. Let $G$ be a finite graph on vertices $V \subset L_{1}^{n}$ in general position, let $E=$ $\left\{e_{i}\right\}_{i \in I_{l}}, l \geq|V|$, be its edges, and let $\mathcal{F}$ be the family of all maps $g: I_{l} \rightarrow L_{1}^{n}$ such that if $e \in E$ is the edge with endpoints $f_{1}$ and $f_{2}$, then $H\left(f_{1}\right) \cap H\left(f_{2}\right) \subseteq H(g(e))$. Then there exists a subset $W \subseteq V(W \neq \emptyset)$ such that for any map $g \in L G P(\mathcal{F})$,

$$
\bigcap_{i=1}^{l} H\left(g\left(e_{i}\right)\right) \subseteq \bigcap_{f \in W} H(f) .
$$

Lemma 3. Let $\left\{V_{i}\right\}_{i=1}^{d}$ be a family of closed subsets of a simplex $S$ in $\mathbb{R}^{d-1}$ with facets $\left\{F_{i}\right\}_{i=1}^{d}$ such that:

1. $V_{i}$ is $F_{i}$-starshaped for all $i \in I_{d}$;

2. $S \subseteq \bigcup_{i=1}^{d} V_{i}$.

Then $\bigcap_{i=1}^{d} V_{i} \neq \varnothing$.

Lemma 4. Let $\left\{U_{i}\right\}_{i=1}^{d}$ be a family of open subsets of simplex $S$ in $\mathbb{R}^{d-1}$ with facets $\left\{F_{i}\right\}_{i=1}^{d}$ such that:

1. $U_{i} \cup \operatorname{rint} F_{i}$ is $\operatorname{rint} F_{i}$-starshaped for all $i \in I_{d}$; 
2. int $S \subseteq \bigcup_{i=1}^{d} U_{i}$;

3. any point $p \in$ bd S has a neighborhood $N(p)$ such that $N(p) \cap \operatorname{int} S \subseteq \bigcup_{i, p \in F_{i}} U_{i}$.

Then $\bigcap_{i=1}^{d} U_{i} \neq \emptyset$.

\section{Reduction of Theorems 1-3 to Special Cases}

We now show that it is sufficient to prove Theorems 1-3 in some special cases.

Lemma 5. It is sufficient to prove Theorems 1-3 for the case of a simple polyhedral set (polytope) X.

Proof. By Lemma 1, $X=S \cap L$, where $S$ is simple polyhedral set, $L$ is an $n$-dimensional affine subspace in $\mathbb{R}^{N}$, and $F_{i}=F_{i}^{S} \cap L$, where $\left\{F_{i}^{S}\right\}_{i \in I_{m}}$ are the facets of $S$. Indeed, Theorems 1-3 for $S$ imply those for $X$, if we note that

$$
\begin{aligned}
& \operatorname{conv}\left(\{a\} \cup F_{i}\right)=L \cap \operatorname{conv}\left(\{a\} \cup F_{i}^{S}\right), \\
& \text { int } \operatorname{conv}\left(A_{i} \cup F_{i}\right)=L \cap \operatorname{int} \operatorname{conv}\left(A_{i} \cup F_{i}^{S}\right), \\
& \text { int } X=L \cap \text { int } S .
\end{aligned}
$$

We now consider a simple polyhedral set $S$, let its facets be $\left\{F_{i}\right\}_{i \in I_{m}}$.

Denote $\mathcal{A}_{1}=\mathbb{R}^{N l}$, the affine space of all possible sets $A$ in Theorem 1 . In Theorems 2 and 3 the spaces of all possible sets $A$ will be some $\mathcal{A}_{2}, \mathcal{A}_{3} \subset \mathcal{A}_{1}$.

Lemma 6. It is sufficient to prove Theorems 1-3 for any everywhere dense subset $\mathcal{B} \subseteq \mathcal{A}_{i}(i=1,2,3)$.

Proof. In fact, let $A \in \mathcal{A}_{i}$, let the elements of $A$ be numbered $a_{1}$ to $a_{l}$.

Take one of Theorems 1-3. Suppose that the sequence $\left\{A^{k}\right\} \subseteq \mathcal{B}$ is such that

$$
A^{k}=\left\{a_{i}^{k}\right\}_{i \in I_{l}}, \quad \forall i \lim _{k} a_{i}^{k}=a_{i} .
$$

Since $\left\{A^{k}\right\} \subseteq \mathcal{B}$, the theorem holds for $A^{k}$. Thus for any $k$ we have a partition of $A^{k}$ into $A_{i}^{k}$.

Obviously, there exists an infinite set $N$ of positive integers such that the partitions of the indices $I_{l}$ corresponding to the partitions of $A^{k}(k \in N)$ coincide. Then

$$
\operatorname{conv}\left(A_{i} \cup F_{i}\right)=\lim _{k} \operatorname{conv}\left(A_{i}^{k} \cup F_{i}\right)
$$

and

$$
\bigcap_{a \in A_{i}} \operatorname{conv}\left(a \cup F_{i}\right)=\lim _{k} \bigcap_{a \in A_{i}^{k}} \operatorname{conv}\left(a \cup F_{i}\right) .
$$

Since the sets $\operatorname{conv}\left(a \cup F_{i}\right)$ are closed, then if

$$
x \notin \bigcup_{i \in I_{m}} \bigcap_{a \in A_{i}} \operatorname{conv}\left(a \cup F_{i}\right),
$$


then if $k$ is large enough

$$
x \notin \bigcup_{i \in I_{m}} \bigcap_{a \in A_{i}^{k}} \operatorname{conv}\left(a \cup F_{i}\right) .
$$

So the case of Theorem 1 is considered.

In case of Theorems 2 and 3 if

$$
x \in \operatorname{int} \operatorname{conv}\left(A_{i} \cup F_{i}\right) \cap \operatorname{int} \operatorname{conv}\left(A_{j} \cup F_{j}\right), \quad i \neq j,
$$

then if $k$ is large enough

$$
x \in \operatorname{int} \operatorname{conv}\left(A_{i}^{k} \cup F_{i}\right) \cap \operatorname{int} \operatorname{conv}\left(A_{j}^{k} \cup F_{j}\right), \quad i \neq j .
$$

In case of Theorem 3, if for some $i$,

$$
\text { int } \operatorname{conv}\left(A_{i} \cup F_{i}\right) \cap \operatorname{int} S \neq \emptyset \text {, }
$$

then for large enough $k$,

$$
\operatorname{int} \operatorname{conv}\left(A_{i}^{k} \cup F_{i}\right) \cap \operatorname{int} S \neq \emptyset \text {. }
$$

Lemma 7. It is sufficient to prove Theorem 1 for the case $A \subset$ int $S$.

Proof. Remember that in this theorem $S$ is a simplex. We construct a map $\pi(x): \mathbb{R}^{n} \rightarrow$ $S$ such that for every $x \in \mathbb{R}^{n}$ and any facet $F_{i}$ of $S$ the following condition holds:

$$
\operatorname{conv}\left(\{x\} \cup F_{i}\right) \supseteq \operatorname{conv}\left(\{\pi(x)\} \cup F_{i}\right) .
$$

For any $x \in S$ put $\pi(x)=x$. Otherwise, put

$$
I=\left\{i \in I_{m}: f_{i}(x) \geq 0\right\}, \quad I^{\prime}=I_{m} \backslash I,
$$

in other words, $\left\{\text { aff } F_{i}\right\}_{i \in I^{\prime}}$ are the hyperplanes that separate $x$ from $S$. Both sets $I$ and $I^{\prime}$ are not empty. Denote $M=\bigcap_{i \in I} F_{i}$ and $N=\bigcap_{i \in I^{\prime}} F_{i}$.

It is clear that $x \notin$ aff $M$ and $x \notin$ aff $N$. Moreover, it can be easily checked that there exists only one pair $\rho(x) \in \operatorname{aff} M, \pi(x) \in \operatorname{aff} N$ such that the points $\{\rho(x), \pi(x), x\}$ lie on the same line in that order.

Note that for every $i \in I, \rho(x) \in F_{i}$ and for every $i \in I^{\prime}, \pi(x) \in F_{i}$, hence for every $i \in I_{m}$ we have $\operatorname{conv}\left(\{x\} \cup F_{i}\right) \supseteq \operatorname{conv}\left(\{\pi(x)\} \cup F_{i}\right)$.

Now Theorem 1 for $A$ and $S$ follows from Theorem 1 for $\pi(A)$ and $S$.

By Lemma 6, if we have to consider the case $A \subset S$, it is sufficient to prove Theorem 1 for the case $A \subset$ int $S$.

By Lemma 6 we may also consider sets $A$ such that $A \in A G P$ and $a \notin$ aff $F_{i}$ for all $a \in A, j \in I_{m}$. The family of sets $A$ such that $A \in A G P$ and $A \cap$ aff $F_{i}=\emptyset$ for all $i$ is open and everywhere dense in $A \in \mathcal{A}_{1}$.

Besides, we may impose another condition. We need some notation to formulate it. 
Let $S$ be a simple polyhedral set, let $\left\{F_{i}\right\}_{i \in I_{m}}$ be its facets, and let $A$ be $A=\left\{a_{i}\right\}_{i \in I_{l}}$. Put by definition

$$
\mathcal{I}=\left\{(i, j, k) \in I_{l} \times I_{m} \times I_{m}: j>k\right\},
$$

denote by $\mathcal{F}(S, A)$ the family of all maps $g: \mathcal{I} \rightarrow L_{1}^{N}$ such that

$$
H(g(i, j, k))=H\left(g_{i j k}\right) \supseteq \operatorname{aff}\left(a_{i}, F_{j} \cap F_{k}\right),
$$

denote by $\mathcal{G}(S)$ the family of all maps $\gamma: \mathcal{I} \rightarrow L_{1}^{N}$ such that

$$
H\left(\gamma_{i j k}\right) \supseteq F_{j} \cap F_{k}, \quad \bigcap_{j>k} H\left(\gamma_{i j k}\right) \neq \emptyset .
$$

Clearly, $\mathcal{F}(S, A) \subseteq \mathcal{G}(S)$ and for any $g \in \mathcal{G}(S)$ there exists $A$ such that $g \in \mathcal{F}(S, A)$.

Thus the family of all sets $A$ such that $\mathcal{F}(S, A) \cap L G P(\mathcal{G}(S)) \neq \emptyset$ is everywhere dense in the variety of all sets $A$.

Thus we will prove Theorems $1-3$ with the following assumptions:

(1) $S$ is a simple polyhedral set with facets $\left\{F_{i}\right\}_{i \in I_{m}}$.

(2) $A \in A G P$ and $a \notin$ aff $F_{i}$ for all $a \in A, i \in I_{m}$.

(3) $\mathcal{F}(S, A)$ contains some $g \in L G P(\mathcal{G}(S))$.

(4) In Theorem 1, $A \subset$ int $S$.

For a given $g \in L G P(\mathcal{G}(S))$ we write $g_{a j k}$ instead of $g_{i j k}$, if the number of elements $a \in A$ equals $i$. Note that under the above conditions

$$
H\left(g_{a j k}\right)=\operatorname{aff}\left(a, F_{j} \cap F_{k}\right),
$$

since

$$
\operatorname{aff}\left(a, F_{j} \cap F_{k}\right) \subseteq H\left(g_{a j k}\right) \quad \text { and } \quad \operatorname{dim}_{a} H\left(g_{a j k}\right)=\operatorname{dim}_{a} \operatorname{aff}\left(a, F_{j} \cap F_{k}\right) .
$$

\section{Proof of Theorems 1-3}

Now we are ready to prove Theorems 1-3.

Proof of Theorem 1. In this theorem $S$ is a simplex. It was noted in the previous section that we only have to consider the case $A \subset$ int $S$.

We use induction on $\operatorname{dim}_{a} S$. In the case $\operatorname{dim}_{a} S=1$ the theorem is obvious.

Denote for all $a \in A$ and $i \in I_{m}$,

$$
V_{a i}=\operatorname{conv}\left(\{a\} \cup \operatorname{rint} F_{i}\right) \backslash\{a\}
$$

and for all $i \in I_{m}$,

$$
U_{i}=\bigcup_{A^{\prime} \subseteq A,\left|A^{\prime}\right|>=l_{i}} \bigcap_{a \in A^{\prime}} V_{a i} .
$$

It is obvious that the sets $U_{i}$ are open and $x \in \operatorname{int} S \cap U_{i}$ iff there are at least $l_{i}$ points $a \in A$ such that $x \in V_{a i}$, in other words, for at least $l_{i}$ points $a \in A$ there exists $y \in \operatorname{rint} F_{i}$ such that $x \in(a y)$. We show that $\left\{U_{i}\right\}_{i \in I_{m}}$ satisfy the conditions of Lemma 4. 
Condition 1 holds. Indeed, let $x \in U_{i}$ and for some $y \in \operatorname{rint} F_{i}, x^{\prime} \in(x y)$. Let $x \in V_{a i}$, then there exists $z \in \operatorname{rint} F_{i}$ such that $x \in(a z)$. Then $\left\langle a x^{\prime}\right\rangle \cap[z y]=z^{\prime} \in \operatorname{rint} F_{i}$ and $x^{\prime} \in\left(a z^{\prime}\right)$.

Thus $x^{\prime} \in V_{a i}$. Considering all $a \in A^{\prime}$ we obtain $x^{\prime} \in U_{i}$.

We now show that condition 3 holds. Let $p \in \operatorname{bd} S, I=\left\{i: p \in F_{i}\right\}$ and $I^{\prime}=I_{m} \backslash I$. Condition 3 holds iff for any $x \in \operatorname{int} S \cap N(p)$ there exists $i \in I$ such that for at least $l_{i}$ points $a \in A$ we have $\langle a x\rangle \cap \operatorname{rint} F_{i} \neq \emptyset$.

For any $a \in A$ the point $y_{a}(x)=\langle a x\rangle \cap$ bd $S$ is a continuous function of $x \in S$. Since $y_{a}(p)=p, a \in A$, there exists a neighborhood $N(p)$ of $p$ such that for any $x \in N(p)$, $a \in A$, and $i \notin I$ we have $y_{a}(x) \notin F_{i}$.

We show that

$$
N(p) \cap \operatorname{int} S \subseteq \bigcup_{i \in I} U_{i}
$$

To prove it we should show that if $x \in N(p) \cap$ int $S$, then for some $i \in I$ we have at least $l_{i}$ points $a \in A$ such that $y_{a}(x) \in \operatorname{rint} F_{i}$. In this case for such $a$ we have $x \in\left(a y_{a}(x)\right)$ and therefore $x \in U_{i}$.

Assume the contrary that for all $i \in I$,

$$
\left|\left\{y_{a}(x)\right\}_{a \in A} \cap \operatorname{rint} F_{i}\right|<l_{i},
$$

or, equivalently,

$$
\left|\left\{y_{a}(x)\right\}_{a \in A} \cap \operatorname{rint} F_{i}\right| \leq l_{i}-1 .
$$

Then for at least $|I|$ points $a \in A$ (denote them by $A^{\prime}$ ) we have

$$
y_{a}(x) \in F_{s(a)} \cap F_{t(a)}, \quad s(a), t(a) \in I .
$$

Hence

$$
x \in \bigcap_{a \in A^{\prime}} \operatorname{aff}\left(a, F_{s(a)} \cap F_{t(a)}\right),
$$

and therefore

$$
\operatorname{aff}\left(a, F_{s(a)} \cap F_{t(a)}\right)=H\left(g_{a s(a) t(a)}\right),
$$

where $g_{\text {ast }}$ is defined above, and

$$
\left\{\text { aff } F_{s(a)}, \text { aff } F_{t(a)}\right\}_{a \in A^{\prime}} \subseteq\left\{H\left(f_{i}\right)\right\}_{i \in I} .
$$

Denote the family of maps

$$
\mathcal{G}^{\prime}=\left\{\gamma: A^{\prime} \rightarrow L_{1}^{N}: H(\gamma(a)) \supseteq F_{s(a)} \cap F_{t(a)}\right\} .
$$

Since the map of restriction $\mathcal{G}(S) \rightarrow \mathcal{G}^{\prime}$ is surjective, the restriction $g^{\prime}: a \mapsto g_{a s(a) t(a)}$ of $g \in L G P(\mathcal{G}(S))$ must be in $L G P\left(\mathcal{G}^{\prime}\right)$.

We apply Lemma 2 to the graph with vertices $\left\{f_{i}\right\}_{i \in I}$, edges $\left(f_{s(a)}, f_{t(a)}\right), a \in A^{\prime}$, and the map $g^{\prime}$. The conditions of the lemma hold since $\left|A^{\prime}\right| \geq|I|$. By Lemma 2 for some $i$ we have

$$
\bigcap_{a \in A^{\prime}} H\left(g_{a}^{\prime}\right) \subseteq H\left(f_{i}\right)=\operatorname{aff} F_{i}
$$


and

$$
x \in \bigcap_{a \in A^{\prime}} H\left(g_{a}^{\prime}\right) \subseteq \operatorname{aff} F_{i}, \quad x \in \text { int } S .
$$

This is a contradiction, hence condition 3 holds.

Consider condition 2.

As above if for some $x \in$ int $S, y_{a}(x) \notin \operatorname{rint} F_{i}$ for all $i \in I_{m}, a \in A$, then

$$
x \in \bigcap_{a \in A} \operatorname{aff}\left(a, \operatorname{aff} F_{s(a)} \cap \operatorname{aff} F_{t(a)}\right) .
$$

Applying Lemma 2 to the graph with vertices $\left\{f_{i}\right\}_{i \in I_{m}}$ and edges $\left(f_{s(a)}, f_{t(a)}\right)(a \in A)$ we obtain that $x \in$ aff $F_{i} \cap$ int $S$ for some $i$. This is a contradiction.

Finally we apply Lemma 4 and obtain $x \in \bigcap_{i \in I_{m}} U_{i}$. Then for any $i \in I_{m}$ there are at least $l_{i}$ points $a \in A$ such that $x \in V_{a i}$.

For any $a \in A$ there exists at most one $i$ such that $x \in V_{a i}$, then for any $i \in I_{m}$ there exists a set $A_{i} \subseteq A$ with $l_{i}$ such that for all $a \in A_{i}, x \in V_{a i}$. The sets $A_{i}$ are obviously disjoint.

Thus we have

$$
x \in \operatorname{conv}\left(\{a\} \cup \operatorname{rint} F_{i}\right) \quad \text { for all } \quad i \in I_{m}, \quad a \in A_{i} .
$$

For all $i \in I_{m}$ and $a \in A_{i}$,

$$
S \subseteq \bigcup_{i \in I_{m}} \operatorname{conv}\left(\{x\} \cup F_{i}\right) \quad \text { and } \quad \operatorname{conv}\left(\{a\} \cup F_{i}\right) \supseteq \operatorname{conv}\left(\{x\} \cup F_{i}\right)
$$

then $S \subseteq \bigcup_{i \in I_{m}} \bigcap_{a \in A_{i}} \operatorname{conv}\left(\{a\} \cup F_{i}\right)$ and the theorem is proved.

Proof of Theorem 2. First consider the case when $S$ is a simplex. Denote

$$
V_{i}=\left\{x \in S: \mid \text { int } \operatorname{conv}\left(\{x\} \cup F_{i}\right) \cap A \mid<l_{i}\right\} .
$$

Obviously, the sets $\left\{V_{i}\right\}_{i \in I_{m}}$ are closed. Also, if $x \in V_{i}, y \in F_{i}$, and $x^{\prime} \in[x y]$, then int conv $\left(\left\{x^{\prime}\right\} \cup F_{i}\right) \subseteq$ int $\operatorname{conv}\left(\{x\} \cup F_{i}\right)$. Hence $V_{i}$ are $F_{i}$-starshaped.

Consider two cases:

Case 1: $S \subseteq \bigcup_{i \in I_{m}} V_{i}$. By Lemma 3 there exists $x \in \bigcap_{i \in I_{m}} V_{i}$. It means that there are at least $m$ points $a \in A$ such that $a \notin$ int $\operatorname{conv}\left(\{x\} \cup F_{i}\right)$ for all $i$, denote the set of such points $a$ by $A^{\prime}$. Then for all $a \in A^{\prime}$,

$$
a \in \operatorname{conv}\left(\{x\} \cup\left(\operatorname{aff} F_{s(a)} \cap \operatorname{aff} F_{t(a)}\right)\right) .
$$

Let $I=\left\{i: x \notin F_{i}\right\}$, then $s(a), t(a) \in I$ for all $a \in A^{\prime}$, otherwise we would have $a \in F_{i}$, which is not true by assumption (2). In other words,

$$
x \in \bigcap_{a \in A^{\prime}} \operatorname{aff}\left(a, \operatorname{aff} F_{s(a)} \cap \operatorname{aff} F_{t(a)}\right) .
$$

As in the proof of Theorem 1 we apply Lemma 2 taking $\left\{f_{i}\right\}_{i \in I}$ as vertices, $\left(f_{s(a)}, f_{t(a)}\right)$, $a \in A^{\prime}$, as edges, and the restriction $g^{\prime}(a)=g_{a s(a) t(a)}$ of the map $g$. By this lemma we have $x \in$ aff $F_{i}, i \in I$, this is a contradiction with the definition of $I$.

Thus case 1 is impossible. 
Case 2: $S \nsubseteq \bigcup_{i \in I_{m}} V_{i} . \quad$ Let $x \in S \backslash \bigcup_{i \in I_{m}} V_{i}$. Then for any $i \in I_{m}$ there exist at least $l_{i}$ points $a \in A$ such that $a \in \operatorname{int} \operatorname{conv}\left(\{x\} \cup F_{i}\right)$.

The sets int $\operatorname{conv}\left(\{x\} \cup F_{i}\right)\left(i \in I_{m}\right)$ do not intersect, hence each of them contains exactly $l_{i}$ points of $A$, so we denote

$$
A_{i}=A \cap \operatorname{int} \operatorname{conv}\left(\{x\} \cup F_{i}\right) .
$$

Then for any $i \in I_{m}$,

$$
\operatorname{conv}\left(A_{i} \cup F_{i}\right) \subseteq \operatorname{conv}\left(\{x\} \cup F_{i}\right),
$$

hence for all $i \neq j \in I_{m}$,

$$
\text { int } \operatorname{conv}\left(A_{i} \cup F_{i}\right) \cap \operatorname{int} \operatorname{conv}\left(A_{j} \cup F_{j}\right)=\emptyset .
$$

Thus the proof is complete if $S$ is a simplex.

Now suppose $S$ is not a simplex, then $\bigcap_{i \in I_{m}} F_{i} \neq \emptyset$. Consider two cases.

Case 1: $\bigcap_{i \in I_{m}} F_{i}=\{v\} . \quad$ Obviously, there exists a hyperplane $H$ such that $S^{\prime}=S \cap H$ is a simplex with facets $F_{i}^{\prime}=F_{i} \cap H, i \in I_{m}$.

Let the central projection from $v$ to $H$ take $a \in A$ to $p(a)$. The theorem is already proved for $A^{\prime}=p(A)$ and simplex $S^{\prime}$, thus there is a partition $A=\bigcup_{i \in I_{m}} A_{i}$ such that $\left|A_{i}\right|=l_{i}$ and for all $i \neq j \in I_{m}$,

$$
\operatorname{int} \operatorname{conv}\left(p\left(A_{i}\right) \cup F_{i}^{\prime}\right) \cap \operatorname{int} \operatorname{conv}\left(p\left(A_{j}\right) \cup F_{j}^{\prime}\right)=\emptyset .
$$

Take the cone $C_{i}=\operatorname{int} \operatorname{conv}\left(F_{i} \cup \bigcup_{a \in A_{i}}[v a\rangle\right)$ formed by the rays from $v$ that intersect int $\operatorname{conv}\left(p\left(A_{i}\right) \cup F_{i}^{\prime}\right)$ without the point $v$. Obviously, we have for all $i \neq j \in I_{m}$,

$$
C_{i} \cap C_{j}=\emptyset
$$

and for all $i \in I_{m}$,

$$
\operatorname{int} \operatorname{conv}\left(A_{i} \cup F_{i}\right) \subseteq \operatorname{int} \operatorname{conv}\left(F_{i} \cup \bigcup_{a \in A_{i}}[v a\rangle\right)=C_{i} .
$$

Then for all $i \neq j \in I_{m}$,

$$
\operatorname{int} \operatorname{conv}\left(A_{i} \cup F_{i}\right) \cap \operatorname{int} \operatorname{conv}\left(A_{j} \cup F_{j}\right)=\emptyset .
$$

In this case the proof is complete.

Case 2: $\operatorname{dim}_{a} L>0$, where $L=\bigcap_{i} F_{i}$. Let $p$ be a projection along $L$. Then $p(L)$ is a point. The previous case gives a partition $A=\bigcup_{i \in I_{m}} A_{i}$ such that for all $i \neq j \in I_{m}$,

$$
\operatorname{int} \operatorname{conv}\left(p\left(A_{i}\right) \cup p\left(F_{i}\right)\right) \cap \operatorname{int} \operatorname{conv}\left(p\left(A_{j}\right) \cup p\left(F_{j}\right)\right)=\emptyset .
$$

Then it is clear that

$$
\text { int } \operatorname{conv}\left(A_{i} \cup F_{i}\right) \cap \operatorname{int} \operatorname{conv}\left(A_{j} \cup F_{j}\right)=\emptyset
$$

and the theorem is proved. 
Proof of Theorem 3. For any $i \in I_{m}$ put

$$
U_{i}=\left\{x \in \operatorname{int} S:[x a] \cap \operatorname{rint} F_{i} \neq \emptyset \text { for at least } l_{i} \text { points } a \in A\right\} .
$$

The sets $U_{i}$ are open.

We show that $\left\{U_{i}\right\}_{i \in I_{m}}$ satisfy the conditions of Lemma 4.

Condition 1 holds. Let for some $a \in A, x \in U_{i}, y \in \operatorname{rint} F_{i}, x^{\prime} \in[x y]$, and $z=[x a] \cap \operatorname{rint} F_{i}$. The points $x^{\prime}$ and $a$ are in different halfspaces with respect to the hyperplane aff $F_{i}$, hence there exists $z^{\prime}=\left[x^{\prime} a\right] \cap$ aff $F_{i}, z^{\prime} \in[z y]$, and $z^{\prime} \in \operatorname{rint} F_{i}$.

Consider condition (3). Let

$$
p \in \operatorname{bd} S, \quad I=\left\{i: p \in F_{i}\right\} .
$$

We prove that there is a neighborhood $N(p)$ of $p$ such that

$$
N(p) \cap \operatorname{int} S \subseteq \bigcup_{i \in I} U_{i}
$$

or, equivalently, for any $x \in \operatorname{int} S \cap N(p)$ for some $i \in I$ at least $l_{i}$ of the segments $\left[x_{j} x\right]$ intersect rint $F_{i}$.

Let $A^{\prime}=\left\{a \in A: a \in \bigcup_{i \in I} H_{i}\right\}$, in this theorem $|A| \geq|I|$ and $[a p] \cap$ bd $S=\{p\}$ for all $a \in A^{\prime}$. Take any $a \in A^{\prime}$ and $x \in S$ and let $y_{a}(x)$ be the farthest point from $x$ in $[a x] \cap$ bd $S$.

Obviously, $y_{a}(x)$ is a continuous function of $x \in S$. Since $y_{a}(p)=p$ for all $a \in A^{\prime}$, then there exists a neighborhood $N(p)$ of $p$ such that for all $x \in N(p), a \in A^{\prime}$, and $i \notin I$ we have $y_{a}(x) \notin F_{i}$.

We shall show that for any $x \in N(p) \cap$ int $S$ there exists $i \in I$ such that $x \in U_{i}$. Equivalently, at least $l_{i}$ of the points $y_{a}(x)\left(a \in A^{\prime}\right)$ are in $\operatorname{rint} F_{i}$ for some $i \in I$.

Assume the contrary. Then we have at least $|I|$ points $a \in A^{\prime}$ such that $y_{a}(x) \in$ $F_{S(a)} \cap F_{t(a)}$ for some $x \in N(p) \cap$ int $S$. Denote the set of these $a$ by $A^{\prime \prime}$. Then

$$
x \in \bigcap_{a \in A^{\prime \prime}} \operatorname{aff}\left(a, \operatorname{aff} F_{s(a)} \cap \operatorname{aff} F_{t(a)}\right), \quad s(a), t(a) \in I .
$$

As in the proof of Theorem 1 by Lemma 2 we get a contradiction, since $\left|A^{\prime \prime}\right| \geq|I|$.

We now show that condition 2 holds. We show that int $S \subseteq \bigcup_{i \in I_{m}} U_{i}$. Assume the contrary: there exists $x$ such that for any $i \in I_{m}$ at least $l_{i}$ of the points $y_{a}(x)$ are in rint $F_{i}$. Denote the set of these points $a$ by $A^{\prime \prime}$. As above we have $x \in \bigcap_{a \in A^{\prime \prime}} \operatorname{aff}\left(a\right.$, aff $F_{s(a)} \cap$ aff $\left.F_{t(a)}\right)$. That is a contradiction, since $\left|A^{\prime \prime}\right| \geq m+1$.

If $S$ is a simplex, then $\bigcap_{i \in I_{m}} U_{i} \neq \emptyset$ by Lemma 4 .

If $\bigcap_{i \in I_{m}} F_{i}=\{v\}$, then as above we take a hyperplane $H$ such that $S^{\prime}=S \cap H$ is a simplex with facets $F_{i}^{\prime}=F_{i} \cap H, i \in I_{m}$. Applying Lemma 4 to $S^{\prime}$ and the sets $U_{i}^{\prime}=U_{i} \cap H$ we obtain

$$
\bigcap_{i \in I_{m}} U_{i}^{\prime} \neq \emptyset \quad \text { and } \bigcap_{i \in I_{m}} U_{i} \neq \emptyset
$$

Take some $x \in \bigcap_{i \in I_{m}} U_{i}$ and put

$$
A_{i}=\left\{a \in A: y_{a}(x) \in \operatorname{rint} F_{i}\right\} .
$$


Then by the definition of $U_{i}$ we have $\left|A_{i}\right| \geq l_{i}$. Also, $\sum_{i \in I_{m}} l_{i}=|A|$ and the sets $A_{i}$ do not intersect pairwise, then $\left|A_{i}\right|=l_{i}$ for all $i \in I_{m}$ and the sets $A_{i}$ form some partition of $A$.

Let $R_{i}$ be a cone of rays $[x y\rangle$, where $y \in \operatorname{rint} F_{i}$ without the point $x . R_{k} \cap R_{l}=\emptyset$ for all $k \neq l \in I_{m}$ and since for all $a \in A_{i}$ we have $\operatorname{rint} F_{i} \ni y_{a}(x) \in[a x]$, then $A_{i} \subset R_{i}$. Hence int $\operatorname{conv}\left(A_{i} \cup F_{i}\right) \subseteq R_{i}$ and

$$
\text { int } \operatorname{conv}\left(A_{k} \cup F_{k}\right) \cap \operatorname{int} \operatorname{conv}\left(A_{l} \cup F_{l}\right)=\emptyset, \quad k \neq l \in I_{m} .
$$

Since $A_{i}$ and $S$ are on different halfspaces with respect to aff $F_{i}$, then for any $i \in I_{m}$,

$$
\text { int } \operatorname{conv}\left(A_{i} \cup F_{i}\right) \cap \operatorname{int} S=\emptyset .
$$

The last case is when $\operatorname{dim}_{a} \bigcap_{i \in I_{m}} F_{i}>0$, let $L=\bigcap_{i \in I_{m}} F_{i}$. As in the previous proof take a projection $p$ along $L$. By the same argument we can deduce the theorem for this case from this theorem for the projection $p(S)$, which is already proved.

\section{Proof of Theorems 4-6 and Corollary 1}

As above we apply Lemma 1 to $X$. Thus we have a simplex $S$ in $\mathbb{R}^{m-1}$ and an $n$ dimensional affine subspace $L \subseteq \mathbb{R}^{m-1}$ such that $X=L \cap S, F_{i}=L \cap G_{i}$, int $X=$ $L \cap \operatorname{int} S$, and int $F_{i}=L \cap \operatorname{int} G_{i}$.

For any point $s \in S$ and $i \in I_{m}$ put $B_{i}(s)=\operatorname{conv}\left(G_{i} \cup\{s\}\right)$. Note that for any $s \in \operatorname{int} S$ the sets $B_{i}(s)$ give a partition of $S$. To prove Theorems 4 and 6 we consider partitions of $X$ into sets $A_{i}=B_{i}(s) \cap L$ for some $s \in$ int $S$. Obviously, $A_{i}(s) \cap$ bd $X=F_{i}$ for any $s \in$ int $S$.

We need a lemma:

Lemma 8. Under the above notation $A_{i}(s)$ are continuous functions of $s \in S$ in the Hausdorff metric, and for any $i \in I_{m}$ and $s \in S, s \notin G_{i}$ we have int $B_{i}(s) \cap L \neq \emptyset$.

Proof. Consider the second statement of the lemma. Assume the contrary:

$$
\text { int } B_{i}(s) \cap L=\emptyset,
$$

then $L \cap$ int $G_{i} \neq \emptyset$ implies that $L \subseteq$ aff $G_{i}$. However, $L \cap G_{j} \neq \emptyset$ for some $j \neq i$ and int $G_{j} \cap$ aff $G_{i}=\emptyset$, that is a contradiction.

Since int $B_{i}(s) \cap L \neq \emptyset$ and $B_{i}(s)$ is a continuous function of $s$, the first statement of the lemma is true for points $s \notin G_{i}$.

For points $s \in G_{i}$ we may prove the continuity by definition: let $s_{l} \rightarrow s$, then, obviously,

$$
\forall l, A_{i}\left(s_{l}\right) \supseteq F_{i}, \quad \lim _{l} A_{i}\left(s_{l}\right)=F_{i}=A_{i}(s)
$$

Now we are ready to prove the theorems. 
Proof of Theorems 4 and 5. Put

$$
U_{i}=\left\{s \in S: \mu\left(A_{i}(s)\right) \leq \mu_{i}\right\} .
$$

We show that Lemma 3 can be applied to $S$ and sets $U_{i}$.

The functions $A_{i}(s)$ and $\mu(A)$ are continuous, then the sets $U_{i}$ are closed.

If $f \in F_{i}, s^{\prime} \in[f s]$, then $A_{i}\left(s^{\prime}\right) \subseteq A_{i}(s)$, and therefore $s^{\prime} \in U_{i}$. It means that the set $U_{i}$ is $F_{i}$-starshaped.

The sets $U_{i}$ cover $S$; otherwise we could find a point $s \in S$ such that for any $i \in I_{m}$, $\mu\left(A_{i}(s)\right)>\mu_{i}$, the sets $A_{i}(s)$ form a partition of $X$, so summing up the inequalities we obtain $\mu(X)>1$, this is a contradiction.

Thus Lemma 3 gives a point $s^{*} \in \bigcap_{i \in I_{m}} U_{i}$. For the partition we have

$$
\mu\left(A_{i}\left(s^{*}\right) \leq \mu_{i},\right.
$$

after summation over $i \in I_{m}$ we have 1 on both sides, hence in fact,

$$
\mu\left(A_{i}\left(s^{*}\right)\right)=\mu_{i} .
$$

Now the proof becomes different for Theorems 4 and 5 .

Case of Theorem 5: Obviously, $A_{i}\left(s^{*}\right) \supseteq F_{i}$ for all $i \in I_{m}$ and if $i \notin I\left(\mu_{i}=0\right)$, then $A_{i}\left(s^{*}\right)=F_{i}$. Hence $X$ is covered by $\left\{A_{i}\left(s^{*}\right)\right\}_{i \in I}$.

Case of Theorem 4: $\mu\left(A_{i}\left(s^{*}\right)\right)>0$ for all $i \in I_{m}$, then $s^{*} \in \operatorname{int} S$ and $A_{i}\left(s^{*}\right) \cap \operatorname{bd} X=$ $F_{i}$ for all $i \in I_{m}$.

Proof of Theorem 6. Put

$$
\left.U_{i}=\left\{s \in S: \operatorname{int} A_{i}(s)\right) \cap V=\emptyset\right\} .
$$

We show that Lemma 3 can be applied to $S$ and the sets $U_{i}$.

If $s \notin U_{i}$, then for some $v \in V$ we have int $A_{i}(s) \ni v$, and for any $s^{\prime}$ in some neighborhood of $s$ we still have int $A_{i}(s) \ni v$. Thus the complement of $U_{i}$ is open and $U_{i}$ is closed.

If $f \in F_{i}$ and $s^{\prime} \in[f s]$, then $A_{i}\left(s^{\prime}\right) \subseteq A_{i}(s)$, and hence $s^{\prime} \in U_{i}$. Thus the set $U_{i}$ is $F_{i}$-starshaped,

The sets $U_{i}$ cover $S$; otherwise we would have a point $s \in S$ such that for any $i \in I_{m}$, $\mid$ int $A_{i}(s) \cap V \mid \geq 1$. The sets $A_{i}(s)$ give a partition of $X$, then $|V| \geq m$, which is a contradiction.

Thus Lemma 3 gives $s^{*} \in \bigcap_{i \in I_{m}} U_{i}$.

We show that we may assume $s^{*} \in$ int $S$. Note that every set $U_{i}$ contains a neighborhood of $G_{i}$. Now let $M$ be a face of $S$ with maximum $\operatorname{dim} M$ and $M \ni s^{*}$, let $M^{\prime}$ be the face spanned by the vertices of $S$ not contained in $M$. Let $m^{\prime} \in \operatorname{rint} M^{\prime}$, then the points $s^{\prime} \in\left(s^{*} m^{\prime}\right)$ close enough to $s^{*}$ will be contained in those of the sets $U_{i}$ that contain $M$ (and therefore a neighborhood of $M$ ). Those sets $U_{i}$ that do not contain $M$, contain $M^{\prime}$, in this case $m^{\prime} \in G_{i}$ and $s^{\prime} \in U_{i}$ due to starshapedness. Thus we have $s^{\prime} \in \operatorname{int} S \cap \bigcap_{i \in I_{m}} U_{i}$.

For the partition we have what we need:

$$
\forall i \in I_{m}, \quad \text { int } A_{i}\left(s^{*}\right) \cap V=\emptyset .
$$


We need another lemma to prove Corollary 1.

Lemma 9. Let $W \subset \mathbb{R}^{n}$ be a finite set in general position, let $|W| \geq n+1$, and let $F$ be a facet of conv $W$. Then there exists a possibly nonconvex polytope with vertices $W$, having $F$ as a facet.

Proof. We use induction over $|W|$. The case $|W|=n+1$ is obvious.

If int conv $W \cap W=\emptyset$, then conv $W$ is the polytope we need.

Otherwise, let $F^{\prime}$ be another facet of conv $W$ and

$$
W^{\prime}=\left(F^{\prime} \cup \operatorname{int} \operatorname{conv} W\right) \cap W .
$$

Clearly, $W^{\prime} \subseteq W$ and $W^{\prime}$ does not contain one of the vertices of $F$, otherwise $F$ and $F^{\prime}$ would coincide. Besides, $F^{\prime} \cap W$ contains at least $n$ points, and int conv $W \cap W$ contains at least one point, hence $\left|W^{\prime}\right| \geq n+1$.

We apply the inductive assumption to $W^{\prime}$ and $F^{\prime}$. We obtain a polytope $Y^{\prime}$ with vertices $W^{\prime}$. Put $Y=\operatorname{conv} W \backslash\left(\operatorname{int} Y^{\prime} \cup \operatorname{rint} F^{\prime}\right)$, this is a polytope, with vertices $W$, having $F$ as a facet.

Proof of Corollary 1. Put $X=\operatorname{conv} W$. Let $\left\{F_{i}\right\}_{i \in I_{m}}$ be the set of facets of $X$. Applying Theorem 6 to $X$ and the set $V$, we obtain a partition of $X$ into sets $A_{i}$ such that $A_{i} \cap \mathrm{bd} X=$ $F_{i}$ and $V \cap$ int $A_{i}=\emptyset$ for all $i \in I_{m}$.

We partition $W \cap \operatorname{int} X$ into sets $W_{i}$ so that $W_{i} \subset A_{i}$ for all $i$. Denote $W_{i}^{\prime}=W \cap F_{i}$.

Consider indices $i \in I_{m}$ such that $W_{i} \neq \emptyset$. Then we apply Lemma 9 to the set $W_{i} \cup W_{i}^{\prime}$ and the facet $F_{i}$, so we have a polytope $Y_{i}$ with vertices $W_{i}$. Note that $Y_{i} \subseteq A_{i}$, therefore int $Y_{i} \cap V=\emptyset$, and $Y_{i} \cap V=\emptyset$ due to general position.

Put $Y=X \backslash\left(\bigcup_{i \in I_{m}, W_{i} \neq \emptyset}\left(\operatorname{int} Y_{i} \cup\right.\right.$ rint $\left.\left.F_{i}\right)\right)$. Then the set of vertices of $Y$ is $W$, and $Y \supset V$, since for all $i \in I_{m}, W_{i} \neq \emptyset$ we have $Y_{i} \cap V=\emptyset$.

\section{Corollary for Partitions of $\mathbb{R}^{n}$}

We should define some properties for a family of closed convex sets $\left\{V_{i}\right\}_{i \in I_{m}}, V_{i} \subseteq L$, where $L$ is a linear space. Some of these properties were already discussed in [2].

Property 1. For any set of $m$ vectors $v_{i} \in L\left(i \in I_{m}\right)$ there exists a permutation $\sigma \in S^{m}$ such that

$$
\bigcup_{i \in I_{m}}\left(V_{i}+v_{\sigma(i)}\right)=L
$$

Property 2. For any set of $m$ vectors $v_{i} \in L\left(i \in I_{m}\right)$ there exists a permutation $\sigma \in S^{m}$ such that the sets

$$
V_{i}^{\prime}=V_{i}+v_{\sigma(i)}
$$

do not intersect pairwise by interior points. 
We also need two definitions:

Definition. A partition of $V \subseteq \mathbb{R}^{n}$ into $V_{i}\left(i \in I_{m}\right)$ is called an affine partition if there are affine functions $\lambda_{i} \in L_{1}^{n}\left(i \in I_{m}\right)$ such that

$$
V_{i}=\left\{x \in V: \forall j \in I_{m} l_{i}(x) \geq l_{j}(x)\right\} .
$$

Definition. A partition of $V \subseteq \mathbb{R}^{n}$ into $V_{i}\left(i \in I_{m}\right)$ is called a hierarchically affine partition if this is either an affine partition or the set $I_{m}$ can be partitioned into subsets $J_{1}, J_{2}, \ldots, J_{k}(k \geq 2)$ such that the sets $V_{j}^{\prime}=\bigcup_{i \in J_{j}} V_{i}\left(j \in I_{k}\right)$ form an affine partition of $V$, and for all $j \in I_{k}$ the sets $V_{i}\left(i \in J_{j}\right)$ form a hierarchically affine partition of $V_{j}^{\prime}$.

We define some stronger variants of Properties 1 and 2 that can be used in recursive proofs for hierarchically affine partitions:

Property 3. For any set of positive integers $l_{i}\left(i \in I_{m}, \sum_{i \in I_{m}} l_{i}=l\right)$ and any set of vectors $A \subset L(|A|=l)$ there exists a partition of $A$ into $A_{i}\left(i \in I_{m}\right)$, satisfying the following conditions:

1. $\left|A_{i}\right|=l_{i}$ for all $i \in I_{m}$.

2. The sets $W_{i}=\bigcap_{a \in A_{i}}\left(V_{i}+a\right)=V_{i} \stackrel{*}{-}\left(-A_{i}\right)\left(i \in I_{m}\right)$ cover $L$, i.e.,

$$
\bigcup_{i \in I_{m}} W_{i}=L
$$

Property 4. For any set of positive integers $l_{i}\left(i \in I_{m}, \sum_{i \in I_{m}} l_{i}=l\right)$ and any set of vectors $A \subset L(|A|=l)$ there exists a partition of $A$ into $A_{i}\left(i \in I_{m}\right)$, satisfying the following conditions:

1. $\left|A_{i}\right|=l_{i}$ for all $i \in I_{m}$.

2. The sets $W_{i}=\operatorname{conv} \bigcup_{a \in A_{i}}\left(V_{i}+a\right)=V_{i}+\operatorname{conv} A_{i}\left(i \in I_{m}\right)$ do not intersect pairwise by interior points, i.e., for all $i \neq j \in I_{m}$,

$$
\text { int } W_{i} \cap \text { int } W_{j}=\emptyset \text {. }
$$

Note that Properties 1 and 2 are contained in Properties 3 and 4, respectively, if we put $l_{i}=1$ for all $i \in I_{m}$.

We formulate a corollary of Theorems 1 and 2:

Corollary 2. Hierarchically affine partitions of $\mathbb{R}^{n}$ have Properties 3 and 4.

We need some lemmas to prove this corollary:

Lemma 10. If a family of closed convex sets $V_{i} \subseteq L$ may be factored modulo some linear subspace $M \subseteq L$, equivalently, $V_{i}=V_{i}+M\left(i \in I_{m}\right)$, then the family of sets $\pi\left(V_{i}\right)$ has Property 1, 2, 3, or 4 in $L / M$, where $\pi: L \rightarrow L / M$ is a projection, iff the family $V_{i} \subseteq L$ has the same property. 
Lemma 11. If a family of closed convex sets $V_{i} \subseteq L$ has one of Properties 1 and 3 in $L$, and for a given subspace $M \subseteq L$ and any $i \in I_{m}, V_{i} \cap M \neq \emptyset$, then the family of sets $V_{i} \cap M$ has the same property in $M$.

Lemma 12. If a family of closed convex sets $V_{i} \subseteq L$ has one of Properties 2 and 4 in $L$, and for a given subspace $M \subseteq L$ and any $i \in I_{m}$ we have $V_{i} \cap M \neq \emptyset$ and $\operatorname{int}\left(V_{i} \cap M\right)=$ (int $\left.V_{i}\right) \cap M \neq \emptyset$, then the family of sets $V_{i} \cap M$ has the same property in $M$.

The proof of these three lemmas is trivial.

Lemma 13. Suppose $V_{i}^{n} \subseteq L\left(i \in I_{m}, n \in \mathbb{Z}^{+}\right)$are closed convex sets. Let for each $i$ the sequence $\left\{V_{i}^{n}\right\}_{n \in \mathbb{Z}^{+}}$converge to a closed convex set $V_{i} \subseteq$ L. If for all $n \in \mathbb{Z}^{+}$the family $\left\{V_{i}^{n}\right\}_{i \in I_{m}}$ has one of Properties 1,2,3, and 4 , then $\left\{V_{i}\right\}_{i \in I_{m}}$ has the same property.

Remark. The limit in the above lemma is considered in the family of metrics

$$
\underset{R}{\operatorname{dist}}(A, B)=\underset{H}{\operatorname{dist}}\left(A \cap B_{0}(R), B \cap B_{0}(R)\right),
$$

where dist ${ }_{H}$ is the Hausdorff metric, and $B_{0}(R)$ is a ball with radius $R$ and center in 0 .

Proof. Consider some sets $A$ and $\left\{l_{i}\right\}_{i \in I_{m}}$. In case of Properties 1 and 2 we simply put $l_{1}=l_{2}=\cdots=l_{m}=1$, so these properties are considered too.

We show that $\left\{V_{i}\right\}_{i \in I_{m}}$ has Property 3 or 4.

Since each family $\left\{V_{i}^{n}\right\}_{i \in I_{m}}$ has the same property, we apply its definition to $A$ and $\left\{l_{i}\right\}_{i \in I_{m}}$.

So for any $n$ we have a partition of $A$, taking some subsequence of families we may assume that the partition of $A$ is the same for all $n$.

Now Property 4 holds because

$$
V_{i}+\operatorname{conv} A_{i}=\lim _{n}\left(V_{i}^{n}+\operatorname{conv} A_{i}\right) .
$$

In the case of Property 3 we may assume the contrary: let there exist a point $x$ such that for any $i \in I_{m}, x \notin V_{i} \stackrel{*}{-}\left(-A_{i}\right)$. Then since the sets $V_{i}$ are closed, we may find $\varepsilon>0$ such that $\operatorname{dist}\left(x, V_{i}+a_{i}\right)>\varepsilon$ for all $i \in I_{m}$ and some $a_{i} \in A_{i}$. It means that for large enough $n$ we have $\operatorname{dist}\left(x, V_{i}+a_{i}\right)>\varepsilon / 2$, and therefore $x \notin V_{i}^{n} \stackrel{*}{-}\left(-A_{i}\right)$ for any $i \in I_{m}$ and large enough $n$. This contradicts the choice of partition $\left\{A_{i}\right\}_{i \in I_{m}}$ in Property 3 for the family $\left\{V_{i}^{n}\right\}_{i \in I_{m}}$.

Proof of Corollary 2. First we consider the following case: the partition of $L$ into $V_{i}$ $\left(i \in I_{m}\right)$ is affine, $m=\operatorname{dim} L+1$, and the system of equations

$$
\lambda_{1}(x)=\lambda_{2}(x)=\cdots=\lambda_{m}(x)
$$

has only one solution, without loss of generality the solution is the origin. In this case $\lambda_{i}$ is a linear function on $L$ for all $i \in I_{m}$. 
For any $i \in I_{m}$ the solution of the system of equations

$$
\forall k, l \neq i, \quad \lambda_{k}(x)=\lambda_{l}(x)
$$

is a straight line. The subset of this line given by the inequality $\lambda_{i}(x)<\lambda_{k}(x)$ for any $k \neq i$ (it is the same for any $k \neq i$ ) is not contained in $V_{i}$, so we choose some vector $s_{i} \neq 0$ from it.

Thus $V_{i}$ is a simplicial cone spanned by the vectors $s_{1}, \ldots, s_{m}$ except $s_{i}$, while vectors $s_{i}$ form a simplex $S$ with facets $F_{i}$ and their respective opposite vertices $\left\{s_{i}\right\}_{i \in I_{m}}$. This simplex contains the origin.

Consider a sequence of positive real numbers $t_{n} \rightarrow \infty$ such that for all $n \in \mathbb{N}$ the homothetic image $t_{n} S \supseteq A$.

So we can apply Theorem 1 or 2 to the set $A$, given numbers $\left\{l_{i}\right\}_{i \in I_{m}}$, and simplex $t_{n} S$. Taking some subsequence of $\left\{t_{n}\right\}$ if needed, we may assume that the partition of $A$ is the same for all $n$.

Note that for all $a \in A, i \in I_{m}$,

$$
V_{i}+a=\lim _{n} \operatorname{conv}\left(\{a\} \cup t_{n} F_{i}\right) .
$$

By going to the limit as in the proof of Lemma 13 we show that Properties 3 and 4 hold in this case.

Now we consider the case of an affine partition into sets $V_{i}$ such that the homogeneous components of degree 1 of the functions $\lambda_{k}-\lambda_{1}(k=2, \ldots, m)$ are linearly independent.

In this case $m \leq \operatorname{dim} L+1$ and the system of equations

$$
\lambda_{1}(x)=\lambda_{2}(x)=\cdots=\lambda_{m}(x)
$$

has an affine subspace $M$ as a solution, applying some translation we may assume that $M$ is a linear subspace. Then $\left\{V_{i}\right\}_{i \in I_{m}}$ may be factored modulo $M$, after the factorization we have $m=\operatorname{dim} L^{\prime}+1\left(L^{\prime}=L / M\right)$ and this case is considered. By Lemma 10 the proof of this case is complete.

Now we consider a more general case: let for all $i \in I_{m}$, int $V_{i} \neq \emptyset$.

If $\lambda_{k}-\lambda_{1}(k=2, \ldots, m)$ are linearly independent, we take some $L^{\prime}=L \oplus \mathbb{R}^{m-1}$ and let the coordinates in the right summand be $x_{2}^{\prime}, \ldots, x_{m}^{\prime}$. Then put $\lambda_{1}^{\prime}=\lambda_{1}, \lambda_{2}^{\prime}=\lambda_{2}+x_{2}^{\prime}$, $\ldots, \lambda_{m}^{\prime}=\lambda_{m}+x_{m}^{\prime}$. The homogeneous components of degree 1 of the functions $\lambda_{k}^{\prime}-\lambda_{1}^{\prime}$ $(k>1)$ are linearly independent. For the affine partition $\left\{V_{i}^{\prime}\right\}_{i \in I_{m}}$ given by the functions $\left\{\lambda_{i}^{\prime}\right\}_{i \in I_{m}}$ we have $V_{i}=L \cap V_{i}^{\prime}$ and int $V_{i}=\left(\right.$ int $\left.V_{i}^{\prime}\right) \cap L \neq \emptyset$. Applying Lemmas 11 and 12 we complete the proof in this case.

Now consider an affine partition such that int $V_{i}=\emptyset$ for some indices $i \in I_{m}$. Let $\left\{\lambda_{i}\right\}_{i \in I_{m}}$ be its affine functions. We show that we can make all int $V_{i}$ nonempty by small changes of $\lambda_{i}$.

Take some positive integer $n$. Consider sets $V_{i}$ such that their $\operatorname{dim} V_{i}$ is minimal, let the set of their indices be $I$. If this dimension is less than $\operatorname{dim} L$, then we can add some number $\varepsilon_{1}<1 / n$ to the functions $\left\{\lambda_{i}\right\}_{i \in I}$ so that the sets $\left\{V_{i}\right\}_{i \in I}$ get some nonempty 
interior, while neither of the $\operatorname{dim} V_{i}$ for $i \notin I$ decrease. The number $\varepsilon_{1}$ can be chosen small enough so that the new sets $\left\{V_{i}\right\}_{i \in I_{m}}$ will be in less than $1 / n$ from their respective old sets in the Hausdorff metric.

We show that the number $\varepsilon_{1}$ can be chosen in more detail. If we choose some points $v_{j} \in \operatorname{rint} V_{j}$ for all $j \notin I$, we see that $\lambda_{i}\left(v_{j}\right)<\lambda_{j}\left(v_{j}\right)$ for all $i \in I, j \notin I$. Hence if $\varepsilon_{1}$ is small enough $\lambda_{i}(i \in I)$ the new sets $V_{j}$ still contain $v_{j}$. If we consider some more points $v_{j} \in V_{j}$, we also show that for small enough $\varepsilon_{1}, \operatorname{dim} V_{j}(j \notin I)$ do not decrease. The fact that the new sets $\left\{V_{i}\right\}_{i \in I}$ have nonempty interiors and for small enough $\varepsilon_{1}$ the Hausdorff distance between new sets $\left\{V_{i}\right\}_{i \in I_{m}}$ and the respective old sets is less than $1 / n$ is obvious.

Applying the above process for no more than $\operatorname{dim} L$ times we may add to some of $\lambda_{i}$ some numbers $\varepsilon_{k}<1 / n$ and finally have $\operatorname{dim} V_{j}=\operatorname{dim} L$, therefore int $V_{j} \neq \emptyset$ for all $j \in I_{m}$. Denote the final partition by $\left\{V_{i}^{n}\right\}_{i \in I_{m}}$.

Each of the partitions $\left\{V_{i}^{n}\right\}_{i \in I_{m}}$ for different $n$ has Properties 3 and 4, so by Lemma 13 the partition $\left\{V_{i}\right\}_{i \in I_{m}}$ has these properties too.

We have considered all cases for affine partitions.

Now we use induction over the number of the sets in a partition to prove the corollary for any hierarchically affine partition.

Consider a set of vectors $A$ and a set of numbers $\left\{l_{i}\right\}_{i \in I_{m}}$. Let $I_{m}$ be partitioned into $J_{j}\left(j \in I_{k}\right)$ and let $V_{j}^{\prime}=\bigcup_{i \in J_{j}} V_{i}$.

We apply the statement of this corollary to the affine partition $\left\{V_{j}^{\prime}\right\}_{j \in I_{k}}$, the set $A$, and the numbers $l_{j}^{\prime}=\sum_{i \in J_{j}} l_{i}$. So the set $A$ can be partitioned into $A_{j}^{\prime}\left(j \in I_{k}\right)$ such that in case of Property 3

$$
\bigcup_{j \in I_{k}} \bigcap_{a \in A_{j}^{\prime}}\left(V_{j}^{\prime}+a\right)=L
$$

or in case of Property 4

$$
\operatorname{int}\left(V_{j_{1}}^{\prime}+\operatorname{conv} A_{j_{1}}^{\prime}\right) \cap \operatorname{int}\left(V_{j_{2}}^{\prime}+\operatorname{conv} A_{j_{2}}^{\prime}\right)=\emptyset, \quad \forall j_{1} \neq j_{2} \in I_{k} .
$$

Now the sets $V_{i}\left(i \in J_{j}\right)$ form a hierarchically affine partition of the set $V_{j}^{\prime}$. Since the partition is given by affine functions, we may consider these functions on the whole $L$ and take $V_{i}^{\prime \prime}\left(i \in J_{j}\right)$ such that $V_{i}=V_{i}^{\prime \prime} \cap V_{j}^{\prime}$ and the sets $V_{i}^{\prime \prime}\left(i \in J_{j}\right)$ give a hierarchically affine partition of $L$.

We apply the inductive assumption to every family $V_{i}^{\prime \prime}\left(i \in J_{j}\right)$, its respective $A_{j}^{\prime}$, and the subset $\left\{l_{i}\right\}_{i \in J_{j}}$. So we obtain partitions of every $A_{j}^{\prime}$ into $A_{i}^{\prime \prime}\left(i \in J_{j}\right)$ such that $\left|A_{i}^{\prime \prime}\right|=l_{i}$, and in the case of Property 3

$$
\bigcup_{i \in J_{j}} \bigcap_{a \in A_{i}^{\prime \prime}}\left(V_{i}^{\prime \prime}+a\right)=L,
$$

or in the case of Property 4

$$
\operatorname{int}\left(V_{i_{1}}^{\prime \prime}+\operatorname{conv} A_{i_{1}}^{\prime \prime}\right) \cap \operatorname{int}\left(V_{i_{2}}^{\prime \prime}+\operatorname{conv} A_{i_{2}}^{\prime \prime}\right)=\emptyset, \quad \forall i_{1} \neq i_{2} \in J_{j} .
$$


Thus in the case of Property 3

$$
\begin{aligned}
\left(\bigcap_{a \in A_{i}^{\prime \prime}}\left(V_{i}+a\right)\right) \cap\left(\bigcap_{a \in A_{j}^{\prime}}\left(V_{j}^{\prime}+a\right)\right) & \\
& =\left(\bigcap_{a \in A_{i}^{\prime \prime}}\left(\left(V_{i}+a\right) \cap\left(V_{j}^{\prime}+a\right)\right)\right) \cap\left(\bigcap_{a \in A_{j}^{\prime}}\left(V_{j}^{\prime}+a\right)\right) \\
& =\left(\bigcap_{a \in A_{i}^{\prime \prime}}\left(V_{i}^{\prime \prime}+a\right)\right) \cap\left(\bigcap_{a \in A_{j}^{\prime}}\left(V_{j}^{\prime}+a\right)\right),
\end{aligned}
$$

and therefore

$$
\bigcup_{i \in J_{j}} \bigcap_{a \in A_{i}^{\prime \prime}}\left(V_{i}+a\right) \supseteq V_{j}^{\prime}
$$

and

$$
\bigcup_{i \in I_{m}} \bigcap_{a \in A_{i}^{\prime \prime}}\left(V_{i}+a\right)=L
$$

It means that Property 3 holds.

For Property 4 for indices $i_{1} \neq i_{2}$ from the same $J_{j}$ we have

$$
\begin{aligned}
\operatorname{int}\left(V_{i_{1}}+\operatorname{conv} A_{i_{1}}^{\prime \prime}\right) & \cap \operatorname{int}\left(V_{i_{2}}+\operatorname{conv} A_{i_{2}}^{\prime \prime}\right) \\
& \subseteq \operatorname{int}\left(V_{i_{1}}^{\prime \prime}+\operatorname{conv} A_{i_{1}}^{\prime \prime}\right) \cap \operatorname{int}\left(V_{i_{2}}^{\prime \prime}+\operatorname{conv} A_{i_{2}}^{\prime \prime}\right)=\emptyset,
\end{aligned}
$$

and for indices $i_{1} \neq i_{2}$ from $J_{j_{1}}$ and $J_{j_{2}}$, respectively,

$$
\begin{aligned}
\operatorname{int}\left(V_{i_{1}}+\operatorname{conv} A_{i_{1}}^{\prime \prime}\right) & \cap \operatorname{int}\left(V_{i_{2}}+\operatorname{conv} A_{i_{2}}^{\prime \prime}\right) \\
& \subseteq \operatorname{int}\left(V_{j_{1}}^{\prime}+\operatorname{conv} A_{j_{1}}^{\prime}\right) \cap \operatorname{int}\left(V_{j_{2}}^{\prime}+\operatorname{conv} A_{j_{2}}^{\prime}\right)=\emptyset .
\end{aligned}
$$

In other words, for any $i_{1} \neq i_{2} \in I_{m}$,

$$
\operatorname{int}\left(V_{i_{1}}+\operatorname{conv} A_{i_{1}}^{\prime \prime}\right) \cap \operatorname{int}\left(V_{i_{2}}+\operatorname{conv} A_{i_{2}}^{\prime \prime}\right)=\emptyset .
$$

Thus Property 4 holds.

In [2] a counterexample was given showing that even in the case of $\mathbb{R}^{3}$ there exist partitions that do not have Properties 1 or 2.

Still it makes sense to search for some more partitions with Properties 1 or 2 . We give some variant of Conjectures 1 and 2 from [2], but before that we need a definition:

Definition. The partition of $\mathbb{R}^{n}$ into closed convex sets $V_{i}\left(i \in I_{m}\right)$ is called ordered with respect to an ordered line $l$ (denote by $\leq_{1}$ the relation on $l$ ) if the family $\left\{V_{i}\right\}$ can be ordered by relation $\leq_{2}$ so that for any translate $l^{\prime}$ of $l$ with translated relation $\leq_{1}$ we have $V_{i} \cap l^{\prime} \leq_{1} V_{j} \cap l^{\prime}$ for all $V_{i} \leq_{2} V_{j}\left(i, j \in I_{m}\right)$. 
Conjecture 1. Any partition of $\mathbb{R}^{n}$ ordered with respect to any ordered line, has Properties 1 and 2 .

As was noted in [2] (Conjectures 3 and 4), the case of $n=2$ of this conjecture is of interest itself because any partition of $\mathbb{R}^{2}$ is ordered with respect to any ordered line.

\section{Acknowledgements}

The author is very grateful to V. L. Dol'nikov for interesting discussions and constant attention to this work. The author also thanks the unknown referees for their detailed comments that helped improve some unclear places in this paper.

\section{References}

1. A. Kaneko and M. Kano, Perfect partitions of convex sets in the plane, Discrete \& Computational Geometry, 28(2) (2002), 211-222.

2. R. N. Karasev, On a conjecture of A. Bezdek, Discrete \& Computational Geometry, 27(3) (2002), 419-439.

3. A. Bezdek, Covering a polygon by triangles with fixed vertices, Geometriae Dedicata, 80(50) (2000), 73-79.

4. A. V. Bogomol'naya, F. L. Nazarov, and S. E. Rukshin, Covering a convex polygon by triangles with given vertices, Matematicheskie Zametki, 43(2) (1988), 191-195.

5. V. V. Proizvolov, Some unsolved problems of combinatorial geometry, Proceedings of the First Soviet Conference on Combinatorial Geometry, Batumi, 1985, p. 54.

Received September 1, 2003, and in revised form February 28, 2004, and September 9, 2004.

Online publication April 15, 2005. 